\title{
Machine Learning based Antenna Selection and Power Allocation in Multi-user MISO Systems
}

\author{
Thang X. Vu, Lei Lei, Symeon Chatzinotas, and Björn Ottersten \\ Interdisciplinary Centre for Security, Reliability and Trust (SnT), University of Luxembourg, Luxembourg \\ Emails: \{thang.vu, lei.lei, symeon.chatzinotas, bjorn.ottersten\}@uni.lu
}

\begin{abstract}
We investigate the performance of multi-user multiple-antenna downlinks via joint antenna selection and power control design. In order to fully exploit the spatial diversity while minimizing the energy consumed by active radio frequency (RF) modules, a subset of antennas are selected to serve the users. Firstly, we propose a joint antenna selection and power allocation (JASPA) algorithm to maximize the system sum rate subjected to the total transmit power constraint and quality of service $(\mathrm{QoS})$ requirements. JASPA copes with the non-convexity of the formulated problem via a doubly iterative algorithm, in which an inner iteration successively optimizes the transmit power followed by an outer loop that tries all valid antenna combinations. Although approaching the global optimality, JASPA suffers a combinatorial complexity, which might limit its application in real-time network operations. To overcome this limitation, we propose a learning-based antenna selection and power allocation (L-ASPA) which significantly reduces the high computational time of JASPA while retaining comparative performance. The core idea behind L-ASPA is to exploit the advances in machine learning to establish underlaying relation between the key system parameters and the selected antennas. The effectiveness of the proposed algorithms is demonstrated via numerical results, which show that JASPA could achieve $90 \%$ of the optimal performance while reducing more than $93 \%$ computation time.
\end{abstract}

Index Terms-Multiuser, Antenna selection, power allocation, machine learning, optimization.

\section{INTRODUCTION}

Multiple-input multiple-output (MIMO) is the key technological enabler to cope with the rapidly increasing demand for data-hungry applications in future mobile networks. Thanks to a large number of antennas, a MIMO base station can send independent information beams to multiple users simultaneously with negligible inter-user interference. The advantages of MIMO, under a proper beamforming design, comprises not only high spectral efficiency and capacity per area but also improved energy efficiency [1]. When the number of antennas in MIMO becomes very large, antenna selection (AS) is employed to improve the MIMO performance in terms of both economical and technological aspects [2]. This is due to the fact that radio frequency (RF) chains are usually much more expensive than antenna elements. More importantly, a proper AS strategy is capable of not only obtaining full spatial diversity but also considerably minimizing the RF chains' energy consumption, hence improving the system energy efficiency [3]-[5]. In general, AS is a NP-hard problem whose optimal solution is only guaranteed via exhausted search, which tries all possibility of antenna combinations [6]. The high complexity of AS might limits its potential in practice, especially in $5 \mathrm{G}$ services which usually requires stringent latency and real-time decision making [7].

Recently, the use of machine learning (ML) in communications systems has attracted much attention [8], [9]. The main advantage of ML-aided communications lays in the capability in establishing underlying relation between system parameters and the desired objective, hence be able to shift the computation burden in real-time processing to the offline training phase. [10], [11]. The authors in [12] proposed a multi-class classification approach to tackle the AS problem in single-user MIMO systems, which were based on two classification methods, namely multiclass k-nearest neighbors and support vector machine (SVM). In [13], a neural networkbased approach was proposed to reduce the computational complexity of AS for multicasting. The neural network (NN) is employed to predict the most potential antenna subset that maximizes the minimum signal to noise ratio among the users. The authors in [14] proposed a learning-based transmit antenna selection to improve the security in wiretap channel. Two learning-based SVM and naive-Bayes schemes were considered. Although being able to improve the secrecy performance with a reduced feedback overhead, [14] is limited to only a single antenna selection.

In this paper, we investigate the joint design for antenna selection and power allocation for multiuser multi-antenna downlinks to improve the energy efficiency while guaranteeing the users' quality of service (QoS) requirements. Our contribution is two-hold. Firstly, we develop a joint antenna selection and power allocation (JASPA) algorithm which relies on the inner approximation method to deal with the nonconvexity of the joint design problem. The proposed JASPA works based on a doubly iterative algorithm, in which an inner iteration successively optimizes the transmit power, followed by an outer iteration that selects the best antenna subset. The convergence of JASPA is theoretically guaranteed. Secondly, in order to overcome the combinatorial complexity of JASPA, which might limit its potential in real-time applications, we propose a machine learning-based antenna selection and power allocation (L-ASPA) algorithm. By exploiting the NN advance, L-ASPA enables underlaying relation between the system parameters and the selected antennas via offline training process. Compared with [12], which studies a single-user MIMO, we consider the multi-user system. Our 
proposed L-ASPA is fundamentally different from [13] since we consider the general unicast, whereas [13] studied the maxmin broadcasting without inter-user interference. Furthermore, our proposed algorithms guarantee user QoS requirements, whereas [13] did not. The effectiveness of the proposed algorithms is demonstrated via numerical results, which show that L-ASPA can achieve more than $90 \%$ the (near) optimal performance while reducing more than $90 \%$ the computational time.

The rest of the paper is organized as follows. Section II presents the system models for downlink multi-antenna systems. Section III presents the proposed JASPA algorithm with quality of service $(\mathrm{QoS})$ constraints. Section IV proposes a L-ASPA algorithm. Numerical results are demonstrated in Section V. Finally, Section IV concludes the paper.

Notations: The superscript $(.)^{T}$ and $(.)^{H}$ stand for the transpose and Hermitian transpose, respectively. $\left(\begin{array}{l}n \\ k\end{array}\right)$ represents the binomial coefficients. $|$.$| and \|$.$\| denote the cardinality and$ the $l_{2}$-norm of a set, respectively.

\section{SySTEM Model}

We consider a downlink multiple-input single-output (MISO) cellular system, in which a base station (BS) equipped with $N$ antennas servers $K$ single-antenna users via a shared wireless medium, where $N>K$. In order to fully exploit spatial diversity gain while minimizing energy consumed by the RF chains, only $M$ antennas are selected to serve the users, with $K \leq M<N$.

Let $\boldsymbol{h}_{k} \in \mathbb{C}^{1 \times N}$ denote the channel vector from the BS's antennas to user $k$, which follows circular-symmetric complex Gaussian distribution $\boldsymbol{h}_{k} \sim \mathcal{C N}\left(\mathbf{0}, \sigma_{k}^{2} \boldsymbol{I}_{N}\right)$, where $\sigma_{k}^{2}$ is the parameter accounting for the path loss from the BS antennas to user $k$. The $\mathrm{BS}$ is assumed to have full channel state information (CSI) to all users. We aim to select a subset of $M$ antennas to serve the users to fully exploit the spatial diversity gain. Denote $\mathcal{A}=\left\{a_{1}, a_{2}, \ldots, a_{M}\right\}, a_{m} \in[N] \triangleq\{1,2, \ldots, N\}$, as a subset of $M$ antennas (out of $N$ ), and denote $\mathcal{A}$ as the collection of all possible antenna subsets. By definition, we have $|\mathcal{A}|=M$ and $|\mathcal{A}|=\left(\begin{array}{l}N \\ M\end{array}\right)$.

Denote $\boldsymbol{h}_{k, \mathcal{A}}$ as the channel vector from all active antennas in a subset $\mathcal{A}$ to user $k$, e.g., $\boldsymbol{h}_{k, \mathcal{A}}=$ $\left[h_{k}\left[a_{1}\right], h_{k}\left[a_{2}\right], \ldots, h_{k}\left[a_{M}\right]\right]$, where $a_{m} \in \mathcal{A}$ and $h_{k}[n]$ is the $n$-th element of $\boldsymbol{h}_{k}$. In order to send data to the users, the BS first applies precoding to suppress inter-user interference. Denote $\boldsymbol{w}_{k, \mathcal{A}} \in \mathbb{C}^{N \times 1}$ as the precoding vector for user $k$ corresponding to the group $\mathcal{A}$. The received signal at user $k$ is given as

$$
y_{k, \mathcal{A}}=\boldsymbol{h}_{k, \mathcal{A}} \boldsymbol{w}_{k, \mathcal{A}} x_{k}+\sum_{i \neq k} \boldsymbol{h}_{k, \mathcal{A}} \boldsymbol{w}_{i, \mathcal{A}} x_{i}+n_{k},
$$

where $n_{k}$ is Gaussian noise with zero mean and variance $\sigma^{2}$. The first term in (1) is the desired signal, and the second term is the inter-user interference.
By treating interference as noise, the achievable data rate of user $k$ is

$$
R_{k}(\mathcal{A})=B \log _{2}\left(1+\frac{\left|\boldsymbol{h}_{k, \mathcal{A}} \boldsymbol{w}_{k, \mathcal{A}}\right|^{2}}{\sum_{i \neq k}\left|\boldsymbol{h}_{k, \mathcal{A}} \boldsymbol{w}_{i, \mathcal{A}}\right|^{2}+\sigma^{2}}\right), \forall k,
$$

where $B$ is the shared channel bandwidth.

In this paper, we employ the minimum mean square error (MMSE) precoding design [15] due to its low complexity. By design, the MMSE precoding vector has a form $\boldsymbol{w}_{k, \mathcal{A}}=$ $\sqrt{p_{k}} \tilde{\boldsymbol{w}}_{k, \mathcal{A}}$, where $p_{k}$ is the power factor allocated to user $k$ and $\tilde{\boldsymbol{w}}_{k, \mathcal{A}}$ is the $k$-th column of the MMSE precoding matrix $\boldsymbol{H}_{\mathcal{A}}^{H}\left(\boldsymbol{H}_{\mathcal{A}} \boldsymbol{H}_{\mathcal{A}}^{H}+\sigma^{2} \boldsymbol{I}_{K}\right)^{-1}$, with $\boldsymbol{H}_{\mathcal{A}}$ is the channel matrix between the active antennas in $\mathcal{A}$ and the users. Denote $\beta_{k, i}^{\mathcal{A}}=$ $\left|\boldsymbol{h}_{k, \mathcal{A}} \tilde{\boldsymbol{w}}_{k, \mathcal{A}}\right|^{2}, \forall i, k$ as the interference factor to user $k$ from the $i$-th user's data stream. By treating interference as noise, the achievable data rate is given as

$$
R_{k}(\mathcal{A})=B \log _{2}\left(1+\frac{\beta_{k, k}^{\mathcal{A}} p_{k}}{\sum_{i \neq k} \beta_{k, i}^{\mathcal{A}} p_{i}+\sigma^{2}}\right), \forall k
$$

The transmit power toward user $k$ is $\lambda_{k} p_{k}$, where $\lambda_{k} \triangleq$ $\left\|\tilde{\boldsymbol{w}}_{k}\right\|^{2}$.

\section{Joint Antenna Selection and Power ALLOCATION}

To fully exploit the spatial diversity while minimizing the RF chains' energy consumption, a subset of $M<L$ antennas are selected to serve the users. In this section, we develop a JASPA algorithm to maximize the system sum rate. The joint optimization problem is formulated as follows:

$$
\begin{aligned}
\mathrm{P} 0: \underset{\mathcal{A} \in \mathcal{A},\left\{p_{k}\right\}_{k=1}^{K}}{\operatorname{Maximize}} & \sum_{k=1}^{K} R_{k}(\mathcal{A}) \\
\text { s.t. } & R_{k}(\mathcal{A}) \geq \eta_{k}, \forall k ; \quad \sum_{k=1}^{K} \lambda_{k} p_{k} \leq P_{\text {tot }},
\end{aligned}
$$

where $R_{k}(\mathcal{A})$ is given in (3), $P_{\text {tot }}$ is the total transmit power, and $\eta_{k}$ is the minimum rate requirement for user $k$.

In general, problem (4) is a mixed binary non-linear problem with binary variable of the activated antennas and positive power control. Because the power allocation is performed for a given selected antenna subset, problem P0 can be reformulated in an iterative form as follows:

$$
\underset{\mathcal{A} \in \mathcal{A}}{\operatorname{Maximize}} \operatorname{P} 1(\mathcal{A}),
$$

where $\mathrm{P} 1(\mathcal{A})$ is the power allocation problem for the candidate antenna subset $\mathcal{A}$, defined as

$$
\begin{aligned}
\mathrm{P} 1(\mathcal{A}): & \operatorname{Max}_{\left\{p_{k}\right\}} B \sum_{k=1}^{K} \log _{2}\left(1+\frac{\beta_{k, k}^{\mathcal{A}} p_{k}}{\sum_{i \neq k} \beta_{k, i}^{\mathcal{A}} p_{i}+\sigma^{2}}\right) \\
\text { s.t. } & B \log _{2}\left(1+\frac{\beta_{k, k}^{\mathcal{A}} p_{k}}{\sum_{i \neq k} \beta_{k, i}^{\mathcal{A}} p_{i}+\sigma^{2}}\right) \geq \eta_{k}, \forall k \\
& \sum_{k=1}^{K} \lambda_{k} p_{k} \leq P_{t o t},
\end{aligned}
$$

where we have used (3) in the objective function.

If problem P1 can be solved optimally, then the optimal solution of P0 can be obtained via an exhausted search in 
(5), which tries all possible antenna subsets. Unfortunately, solving problem $\mathrm{P} 1$ is challenging due to the non-convexity of the objective function.

By introducing arbitrary positive variables $\left(x_{k}\right)_{k=1}^{K}$, we can reformulate problem $\mathrm{P} 1$ as follows:

$$
\begin{aligned}
\underset{\left(p_{k}, x_{k}\right)_{k=1}^{K}}{\operatorname{Maximize}} & B \sum_{k=1}^{K} x_{k} \\
\text { s.t. } & \log _{2}\left(1+\frac{\beta_{k, k}^{\mathcal{A}} p_{k}}{\sum_{i \neq k} \beta_{k, i}^{\mathcal{A}} p_{i}+\sigma^{2}}\right) \geq x_{k}, \forall k \\
& (6 \mathrm{~b}) ; x_{k} \geq \eta_{k} / B, \forall k,
\end{aligned}
$$

where the second constraint is to guarantee the QoS.

Although the objective function of (7) is now convex, solving problem (7) is still challenging due to the non-convexity of constraint (7a). By defining parameters $A_{k}=\left[\beta_{k, 1}^{\mathcal{A}}, \beta_{k, 2}^{\mathcal{A}}, \ldots, \beta_{k, K}^{\mathcal{A}}\right]$, $B_{k}=\left[\beta_{k, 1}^{\mathcal{A}}, \ldots, \beta_{k, k-1}^{\mathcal{A}}, 0, \beta_{k, k+1}^{\mathcal{A}}, \ldots, \beta_{k, K}^{\mathcal{A}}\right]$, and introducing intermediate variables $\left(y_{k}\right)_{k=1}^{K}$, constraint (7a) is equivalent to

$$
\begin{aligned}
\log \left(A_{k} \boldsymbol{p}+\sigma^{2}\right) & \geq \log (2) x_{k}+y_{k}, \forall k \\
B_{k} \boldsymbol{p}+\sigma^{2} & \leq e^{y_{k}}, \forall k
\end{aligned}
$$

where $\boldsymbol{p} \triangleq\left[p_{1}, \ldots, p_{K}\right]^{T}$ denotes the power allocation vector for all users. We observe that constraint (8a) is convex. However, since the function exp is convex, constraint $(8 b)$ is unbounded. To overcome this difficulty, we employ the inner approximation method, which uses the first-order approximation of $e^{y_{k}}$ at the right hand side of (8b). The approximated problem of (7) is formulated as follows:

$$
\begin{aligned}
\mathrm{P} 2\left(\boldsymbol{y}_{0}\right): & \underset{\boldsymbol{p},\left(x_{k}, y_{k}\right)_{k=1}^{K}}{\operatorname{maximize}} B \sum_{k=1}^{K} x_{k} \\
\text { s.t. } & \log \left(A_{k} \boldsymbol{p}+\sigma^{2}\right) \geq \log (2) x_{k}+y_{k}, \forall k \\
& B_{k} \boldsymbol{p}+\sigma^{2} \leq e^{y_{0 k}}\left(y_{k}-y_{0 k}+1\right), \forall k \\
& (7 \mathrm{~b}),
\end{aligned}
$$

where $\boldsymbol{y}_{0} \triangleq\left(y_{0 k}\right)_{k=1}^{K}$ is any feasible point of constraint (8b).

It is evident that, for a given $\boldsymbol{y}_{0}$, problem (9) is convex since the objective function and the constraints are convex. Thus, it can be solved in an efficient manner by standard solvers, e.g., CVX. Because $e^{a}(x-a+1) \leq e^{x}, \forall a$, the approximated problem (9) always gives a suboptimal solution of the original problem (7).

It is worth noting that the optimal solution of problem (9) is largely determined by parameters $\left(y_{0 k}\right)_{k=1}^{K}$. Thus, it is crucial to select proper values $\left(y_{0 k}\right)_{k=1}^{K}$ such that the solution of (9)

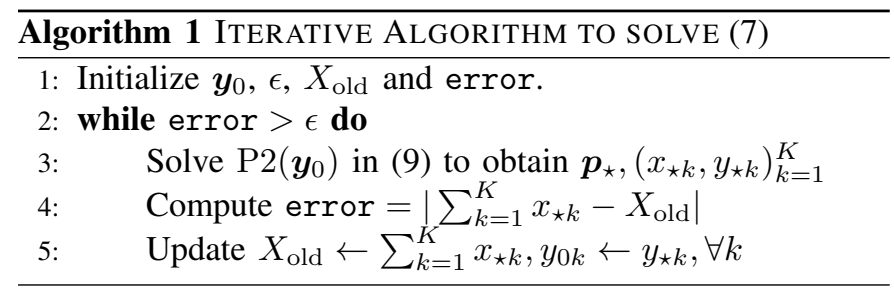

is close to the optimal solution of (7). As such, we propose an iterative optimization algorithm to improve the performance of problem (9) as shown in Algorithm 1. The premise behind the proposed algorithm is to better select the parameters $\left(y_{0 k}\right)_{k=1}^{K}$ through iterations.

Proposition 1 (Convergence of Algorithm 1): The objective function of problem P2( $\left.\boldsymbol{y}_{0}\right)$ in (9) solved by Algorithm 1 decreases by iterations.

The proof of Proposition 1 is shown in Appendix A. Although not guaranteeing the global optimum of problem (7), Proposition 1 justifies the convergence of the proposed iterative algorithm.

Combining (5) and (9), we can solve the original joint antenna selection and power allocation problem (4) via a doubly iterative algorithm, as shown in Algorithm 2.

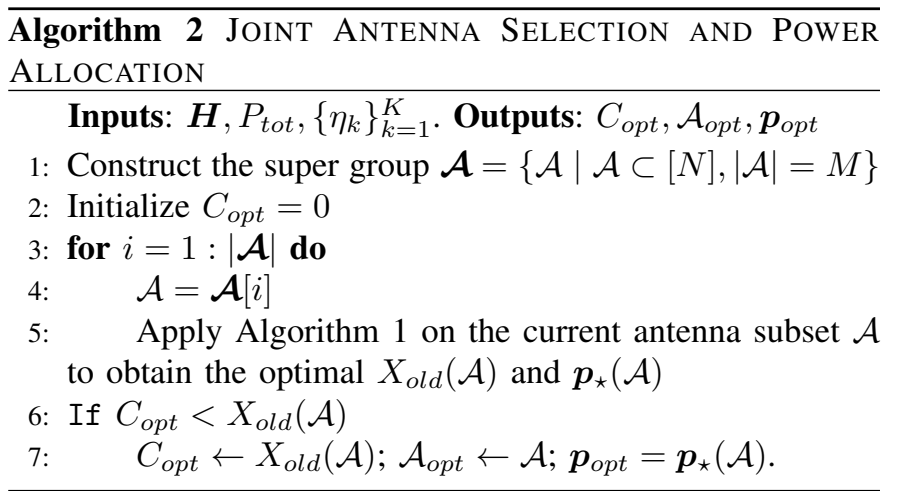

\section{A. Complexity analysis for JASPA}

The proposed JASPA algorithm consists of two iterations. The outer iteration tries all possibilities for the subset of $M$ antennas, and the inner iteration solves optimize the transmit power iteratively. While the complexity of the inner iteration is relatively reasonable since problem (9) is convex, the outer iteration's complexity increases combinatorially with the number of antennas. In fact, the JASPA has to go through all $\left(\begin{array}{l}N \\ M\end{array}\right)$ candidates for the selected antennas. As an example, for $L=20, N=8$, the JASPA examines 125970 possible antenna subsets, each of which imposes an inner loop in Algorithm 1. Although JASPA approaches the optimal performance, the high computation complexity might limit its capability in practical scenarios, especially in the $5 \mathrm{G}$ applications with stringent latency requirements.

\section{Learning-Based Antenna Selection and Power ALLOCATION}

In this section, we propose a learning-based antenna selection and power allocation (L-ASPA) algorithm which overcomes the high computational complexity of JASPA. The premise behind L-ASPA is to exploit machine-learning based predictions to help the optimal algorithm to tackle the most difficult and time-consuming part in the optimization. In particular, L-ASPA will first predict potential subsets of antennas, which will be much smaller than $\left(\begin{array}{l}N \\ M\end{array}\right)$. 
We deploy NN as the learning model to establish underlaying relation between the system parameters (inputs) and the selected antenna subset. The NN consists of three main parts: one input layer, one output layer and one (or several) hidden layer. Based on the labeled data, the NN will optimize the learning parameters in order to minimize the prediction error, e.g., cost function. The L-ASPA is implemented via 3 steps: i) training data generation, ii) building the learning model, and iii) real-time prediction.

1) Training data generation: It is crucial to determine features which will be extracted from the CSI. Because the NN accepts only real-value inputs, the original complex representation of the channel matrix is invalid. One important observation is that the performance of problem (4) heavily depends on inter-user interference, which is determined by crossproduct between the channel vector between any pair of users. Therefore, we choose $\boldsymbol{v}=\operatorname{abs}\left(\operatorname{vec}\left(\boldsymbol{H}^{H} \boldsymbol{H}\right)\right)^{2} \in \mathbb{R}^{K N \times 1}$ as the training input. We note that user location, i.e., pathloss, is already captured in $\boldsymbol{v}$.

Once the input sample is given, we need to define the output, which is the selected antenna combination that provides the maximum objective function in (4). For each training input $\boldsymbol{v}$, we define an output vector $\boldsymbol{b} \in\{0,1\}^{\left(\begin{array}{l}N \\ M\end{array}\right) \times 1}$ indicating the selected antenna subset, e.g., $b[n]=1$ if the $n$-th subset is selected, otherwise $b[n]=0$. Because we are interested in selecting only one subset, we have $\|\boldsymbol{b}\|_{0}=1$ In order to compute $\boldsymbol{b}$, for each channel realization $\boldsymbol{H}$ (corresponding to $\boldsymbol{v}$ ), we run the optimization (5) to find the selected antenna subset $\mathcal{A}^{\star}$ and then assign the output element $b\left[n^{\star}\right]=1$ corresponding to $\mathcal{A}^{\star}$.

Denote $N_{S}$ as the number of samples used to train the learning model. The total training input is aggregated in the input matrix $\boldsymbol{X}=\left[\boldsymbol{v}_{1}, \boldsymbol{v}_{2}, \ldots, \boldsymbol{v}_{N_{S}}\right]$, where $\boldsymbol{v}_{t}$ is the $t$-th input sample. Similarly, the training output matrix is $\boldsymbol{B}=\left[\boldsymbol{b}_{1}, \ldots, \boldsymbol{b}_{N_{S}}\right]$, where $\boldsymbol{b}_{t}$ is the $t$-th output sample corresponding to the input sample $\boldsymbol{v}_{t}$.

2) Building the learning model: When the training data are available, they will be used to train the NN with the learning parameter $\boldsymbol{\Theta}$. For a $L$-layer $\mathrm{NN}$, we have $\boldsymbol{\Theta}=\left[\boldsymbol{\theta}_{1}, \ldots, \boldsymbol{\theta}_{L}\right]$, where $\boldsymbol{\theta}_{l} \in \mathbb{R}^{N_{l} \times 1}, 1 \leq l \leq L$, is the learning parameters in the $l$-th layer. We employ the sigmoi function, $\operatorname{sigmoi}(z)=$ $\left(1+e^{-z}\right)^{-1}$, as the activator of the NN. The learning phase can be done via the minimization of prediction error

$$
\begin{aligned}
\Delta(\boldsymbol{\Theta}) & =\frac{1}{N_{S}} \|-\operatorname{Tr}\left(\boldsymbol{B}^{T} \log \left(f_{\boldsymbol{\Theta}}(\boldsymbol{X})\right)\right) \\
& -\operatorname{Tr}\left(\overline{\boldsymbol{B}}^{T} \log \left(1-f_{\boldsymbol{\Theta}}(\boldsymbol{X})\right)\right)\left\|^{2}+\frac{\lambda}{2 N_{S}} \sum_{l=1}^{L}\right\| \boldsymbol{\theta}_{l} \|^{2},
\end{aligned}
$$

where $\lambda$ is the regulation parameter, $\bar{B}=\mathbf{1}-\boldsymbol{B}$, and $f_{\Theta}(\boldsymbol{X})$ is the prediction of the output layer.

3) Real-time prediction: When the $\mathrm{NN}$ has been well trained, it is ready to provide real-time and highly accurate predictions. From the current channel coefficient matrix $\boldsymbol{H}$, we construct the input of the NN $\boldsymbol{v}=\operatorname{abs}\left(\operatorname{vec}\left(\boldsymbol{H}^{H} \boldsymbol{H}\right)\right)^{2}$. Then $\boldsymbol{v}$ is used as the input of the well-trained NN to output the prediction vector $\hat{b}$, from which the best antenna subset is announced. In general, the larger an element in $\hat{b}$ is, the higher chance this element corresponds to the best antenna subset. It is worth noting that the NN does not provide absolute prediction, e.g., 0 or 1 , but probabilistic uncertainties, e.g., $0 \leq \hat{b}[n] \leq 1, \forall n$. Given the predicted vector $\hat{\boldsymbol{b}}$, we select $K_{S}$ antenna subsets corresponding to the $K_{S}$ largest elements in $\hat{\boldsymbol{b}}$. Then we apply the power optimization algorithm on these $K_{S}$ subsets instead of $\left(\begin{array}{l}N \\ M\end{array}\right)$. The steps of L-ASPA algorithm are listed in Algorithm 3. Compared with JASPA, L-ASPA significantly reduces the computational time since it tries only $K_{S}$ promising candidates while JASPA examines all over $\left(\begin{array}{l}N \\ M\end{array}\right) \gg K_{S}$ antenna combinations.

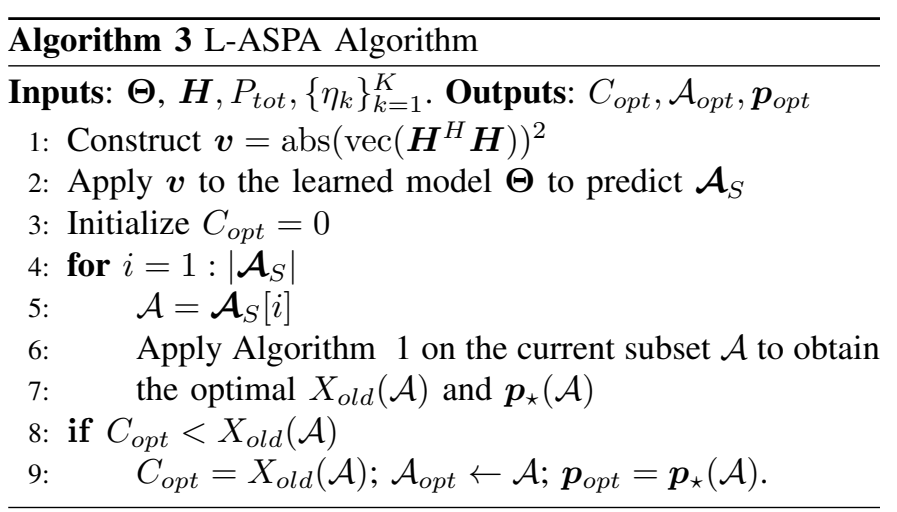

\section{Performance Evaluation}

In this section, we evaluate the performance of the proposed JASPA and L-ASPA algorithms via numerical results. All wireless channels are subject to Rayleigh fading. The users are uniformly distributed in an area between 50 and 100 meters from the BS. With a pathloss exponent equal to 3.5, the pathloss is uniformly distributed between $-59.4 \mathrm{~dB}$ and $-70 \mathrm{~dB}$. The total antennas number is $N=8$, the channel bandwidth $B=1 \mathrm{MHz}, P_{t o t}=10 \mathrm{~W}$, and the minimum rate requirements is $\eta_{k}=0.1 \mathrm{Mbps}, \forall k$. The number of active antennas is equal to number of users, i.e., $M=K$.

\section{A. Performance-complexity trade-off of L-ASPA}

In this subsection, we examine the efficiency of the proposed L-ASPA via a performance-complexity gain trade-off. By confining the search space of the prediction output, i.e., $K_{\mathcal{S}}$ - the number of potential antenna subsets, we can manage the complexity of L-ASPA since it will work only on $K_{\mathcal{S}}$ candidates. The complexity gain of L-ASPA is defined as the ratio between the computational time reduction divided by the JASPA's computational time, calculated as:

$$
\theta\left(K_{\mathcal{S}}\right)=\frac{\tau\left(\left(\begin{array}{l}
N \\
M
\end{array}\right)-K_{\mathcal{S}}\right)}{\tau\left(\begin{array}{l}
N \\
M
\end{array}\right)}=1-\frac{K_{\mathcal{S}}}{\left(\begin{array}{l}
N \\
M
\end{array}\right)},
$$

where $\tau$ is the computational time for optimizing the power control of a given antenna combination, shown in Algorithm 1. The performance is defined as the ratio between the sum rate obtained by L-ASPA divided by the optimal sum rate of the JASPA algorithm. 


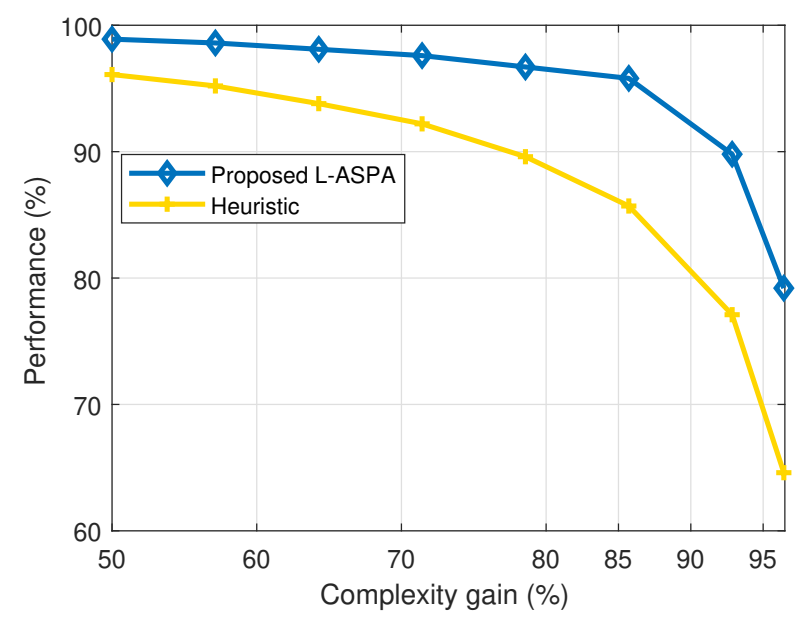

Figure 1. Performance-complexity tradeoff of the proposed L-ASPA.

Fig. 1 plots the performance-complexity tradeoff of the proposed L-ASPA when serving 2 users. It is observed that L-ASPA retains more than $96 \%$ the optimal sum rate while saving more than $85 \%$ complexity. Even when spending only $7 \%$ the computational time, L-ASPA still achieves $90 \%$ the optimal performance, which confirms the effectiveness of the proposed L-ASPA algorithm. Compared with the heuristic solution, L-ASPA further reduces $15 \%$ the computational time at the $90 \%$ performance gain target.

Fig. 2 plots the relative performance in the real-time prediction of L-ASPA versus the number of training samples. The relative performance is measured as the ratio of the L-ASPA's sum rate divided by the one obtained by JASPA. Each training sample is generated randomly and captures the randomness in both channel small-scale fading and user location. In general, having more training samples results in better prediction accuracy since the L-ASPA learn more about the intrinsic relation between the selected antennas and the input features. It is shown that 2000 training samples are sufficient for LASPA to achieve $90 \%$ the optimal performance.

\section{B. Performance comparison}

The performance of the proposed JASPA and L-ASPA algorithms are shown in Fig. 3 for various number of users. In addition, two schemes Heuristic and Equal power are also included for comparison. The Heuristic scheme also performs power allocation but it searches for the selected antennas heuristically. Whereas in the Equal power scheme, the best antenna subset is selected in a similar manner as in L-ASPA but without power allocation. The value of $K_{\mathcal{S}}$ in L-ASPA is chosen such that it imposes only $10 \%$ complexity of the JASPA algorithm. We note that the schemes in [13], [14] are not applicable in our setup since [13] designs only one broadcasting precoding vector for all users, while [14] selects only a single antenna.

Fig. 3 shows that the JASPA provides the largest sum rate since it achieves the (near) optimal performance. It is

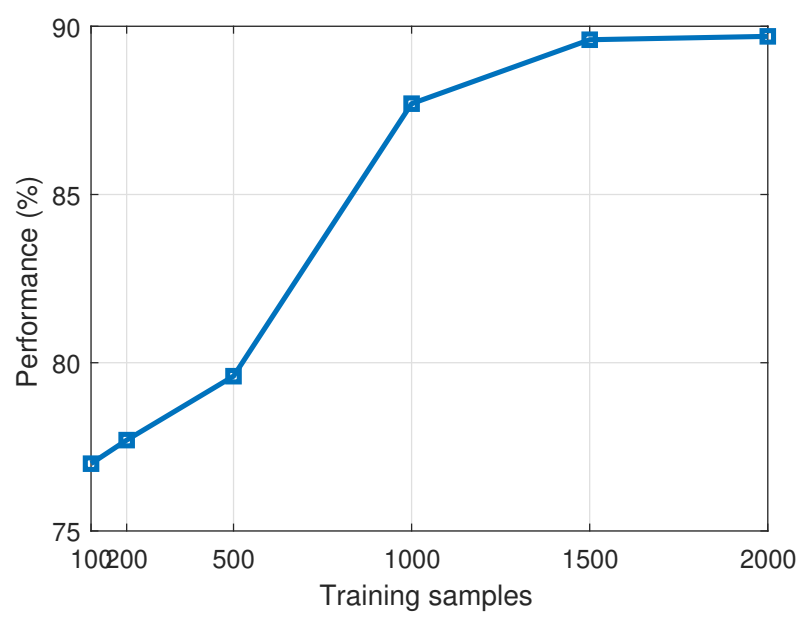

Figure 2. Learning performance versus the number of training samples. Two antenna are selected. Complexity gain is equal to $93 \%$.

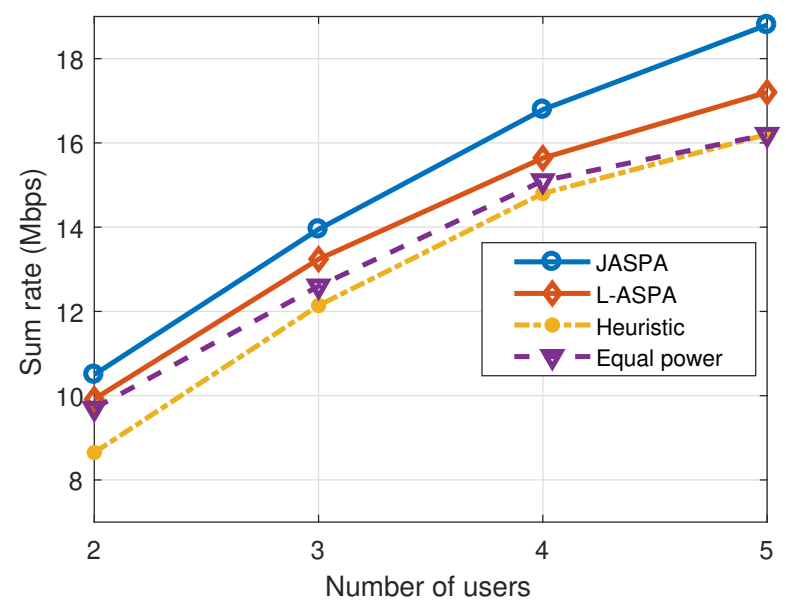

Figure 3. Performance of the proposed algorithms versus the number of users. The number of selected antennas is equal to the user number.

worth noting that JASPA's performance is the best we can obtain because of the non-convexity of the original problem (6). By applying ML to predict potential antenna candidates, the L-ASPA algorithm significantly reduces the computational time while retaining more than $92 \%$ of the (near) optimal performance. In particular, the L-ASPA achieves $94 \%$ and $92 \%$ of the (near) optimal sum rate for $K=2$ and $K=5$, respectively. Compared with the two reference schemes, the proposed L-ASPA outperforms for all parameters. This clearly confirms the effectiveness of the proposed L-ASPA in minimizing the computational overhead. One interesting observation is that the equal power scheme obtains a close performance as L-ASPA for small $K$. This is because the BS has plenty power budget to serve all users for small $K$, hence the optimal power allocation approaches the uniform distribution. When $K$ increase, the system resource becomes scarce, therefore the optimal transmit power is no longer close to equal power allocation, which is 
seen from a big gap between L-ASPA and the equal power curve for large $K$.

The performance of L-ASPA can be further improved by allowing a larger searching space, i.e., larger $K_{\mathcal{S}}$, in Algorithm 3, which however imposes more computational time in L-ASPA.

\section{CONCLUSIONS}

We studied joint design for antenna selection and power allocation in multiuser downlinks to minimize the RF chain energy consumption while fully exploiting the spacial diversity. We first proposed a joint antenna selection and power allocation algorithm (JASPA), which is based on the inner approximation to overcome the non-convexity of the formulated problem. In order to high complexity due to the double iteration structure of JASPA, we then developed a machine learning-based solution to provide appropriate and time-stringent antenna predictions. We showed via numerical results that the learning-based solution can significantly reduce the execution time of JASPA while retaining more than $90 \%$ the optimal performance.

\section{ACKNOWLEDGMENTS}

The work is supported by the project FNR CORE ProCAST, grant C17/IS/11691338, project FNR CORE ROSETTA, grant 11632107 , and by the ERC AGNOSTIC project under code R-AGR-3283.

\section{APPENDIX A}

PROOF OF PROPOSITION 1

Denote $\left(\boldsymbol{p}_{\star}^{(t)},\left\{x_{\star k}^{(t)}, y_{\star k}^{(t)}\right\}_{k=1}^{K}\right)$ as the optimal solution of $\mathrm{P} 2\left(\boldsymbol{y}_{0}^{(t)}\right)$ at iteration $t$. We will show that if $y_{\star k}^{(t)}<y_{0 k}^{(t)}, \forall k$, then by using $y_{0 k}^{(t+1)}=y_{\star k}^{(t)}$ in the $(t+1)$-th iteration, we will have $\sum_{k} x_{\star k}^{(t+1)}>\sum_{k} x_{\star k}^{(t)}$, where $\left\{x_{\star k}^{(t+1)}\right\}_{k=1}^{K}$ is the solution at iteration $t+1$. Indeed, by choosing a relatively large initial value $\boldsymbol{y}_{0}^{(1)}$, we always have $y_{\star k}^{(1)}<y_{0 k}^{(1)}, \forall k$.

Denote $f(x ; a)=e^{a}(x-a+1)$ as the first order approximation of the $e^{x}$ function at $a$. At iteration $t+1$, we have $y_{0 k}^{(t+1)}=y_{\star k}^{(t)}, \forall k$. Therefore, $f\left(y ; y_{\star k}^{(t)}\right)$ is used in the right-hand side of constraint $(9 \mathrm{~b})$ at the $(t+1)$-th iteration. Consider a candidate $\left(y_{1}^{(t+1)}, \ldots, y_{K}^{(t+1)}\right)$ for any $y_{k}^{(t+1)} \in$ $\left(\hat{y}_{k}, y_{\star k}^{(t)}\right)$, where $\hat{y}_{k}=y_{\star k}^{(t)}-1+e^{y_{0 k}^{(t)}-y_{\star k}^{(t)}}\left(y_{\star k}^{(t)}-y_{0 k}^{(t)}+1\right)$. Because function $e^{x}$ is convex and $y_{k}^{(t+1)}<y_{\star k}^{(t)}$, then we have $f\left(y_{k}^{(t+1)} ; y_{\star k}^{(t)}\right)>f\left(y_{\star k}^{(t)} ; y_{0 k}^{(t)}\right), \forall k$. Therefore, there exits $p_{k}^{(t+1)}>p_{\star k}^{(t)}$ and $x_{k}^{(t+1)}>x_{\star k}^{(t)}$ which satisfies constraints (9a) and (9b). Consider a new set $\left\{p_{k}^{(t+1)}, x_{k}^{(t+1)}, y_{k}^{(t+1)}\right\}_{k=1}^{K}$. This set satisfies all the constraints of problem P2 $\left(\boldsymbol{y}_{\star}^{(t)}\right)$, and therefore is a feasible solution of the optimization problem. As the result, the optimal objective at iteration $(t+1), \sum_{k} x_{\star k}^{(t+1)}$, must satisfy $\sum_{k} x_{\star k}^{(t+1)} \geq \sum_{k} x_{k}^{(t+1)}>\sum_{k} x_{\star k}^{(t)}$, which completes the proof of Proposition 1.

\section{REFERENCES}

[1] E. G. Larsson, O. Edfors, F. Tufvesson, and T. L. Marzetta, "Massive MIMO for next generation wireless systems," IEEE Commun. Mag. vol. 52, no. 2, pp. 186-195, Feb. 2014.

[2] R. Heath and A. Paulraj, "Antenna selection for spatial multiplexing systems based on minimum error rate," in IEEE Int. Conf. Commun., Jun. 2001, pp. 2276-2280.

[3] Y. Pei, T.-H. Pham, and Y. Liang, "How many RF chains are optimal for large-scale MIMO systems when circuit power is considered?" in Proc. IEEE Global Commun. Conf., Dec. 2012, pp. 3868-3873.

[4] O. Tervo, L. Tran, H. Pennanen, S. Chatzinotas, M. Juntti, B. Ottersten, "Energy-Efficient Multi-Cell Multigroup Multicasting with Joint Beamforming and Antenna Selection,' IEEE Trans. Signal Process., vol. 66, no. 18 , pp. 4904-4919, 2018.

[5] S. Domouchtsidis, C. Tsinos, S. Chatzinotas, and B. Ottersten, "SymbolLevel Precoding for Low Complexity Transmitter Architectures in LargeScale Antenna Array Systems,' IEEE Trans. Wireless Commun., vol. 18, no. 2, pp. 852-863, Feb. 2019.

[6] O. Mehanna, N. D. Sidiropoulos, and G. B. Giannakis, "Multicast beamforming with antenna selection," in IEEE Int. Workshop Signal Process. Adv. Wireless Commun., Jun. 2012, pp. 70-74.

[7] J. G. Andrews, S. Buzzi, W. Choi, S. V. Hanly, A. Lozano, A. C. K. Soong, and J. C. Zhang, "What will 5G be?" IEEE J. Sel. Areas Commun., vol. 32, no. 6, pp. 1065-1082, Jun. 2014.

[8] L. Lei, L. You, G. Dai, T. X. Vu, D. Yuan, and S. Chatzinotas, "A deep learning approach for optimizing content delivering in cache-enabled HetNet," in Proc. IEEE Int. Symp. Wireless Commun. Syst., Aug. 2017, pp. 449-453.

[9] T. OShea and J. Hoydis, "An introduction to deep learning for the physical layer," IEEE Trans. Cog. Commun. Netw., vol. 3, no. 4, pp. 563-575, Dec. 2017.

[10] H. Sun, X. Chen, Q. Shi, M. Hong, X. Fu, and N. D. Sidiropoulos, "Learning to optimize: Training deep neural networks for wireless resource management," in IEEE Int. Workshop Signal Process. Adv. Wireless Commun., Jul. 2017, pp. 247-252.

[11] L. Lei, T. X. Vu, L. You, S. Fowler, and D. Yuan, "Efficient minimumenergy scheduling with machine-learning based predictions for multiuser MISO systems," in Proc. IEEE Int. Conf. Commun., May 2018, pp. 1-6.

[12] J. Joung, "Machine Learning-Based Antenna Selection in Wireless Communications," in IEEE Commun. Lett., vol. 20, no. 11, pp. 22412244, Nov. 2016.

[13] M. S. Ibrahim, A. S. Zamzam, X. Fu, and N. D. Sidiropoulos, "Learningbased antenna selection for multicasting," in Proc. IEEE Int. Workshop Signal Process. Adv. Wireless Commun., Jun. 2018, pp. 1-5.

[14] D. He, C. Liu, T. Q. S. Quek, and H. Wang, "Transmit antenna selection in MIMO wiretap channels: A machine learning approach," IEEE Wireless Commun. Lett., vol. 7, no. 4, pp. 634-637, Aug. 2018.

[15] S. Kaviani, O. Simeone, W. A. Krzymien and S. Shamai, "Linear MMSE precoding and equalization for network MIMO with partial cooperation," in Proc. IEEE Global Commun. Conf., Dec. 2011, pp. 1-6. 